\title{
Innovative Design of an Elliptical Trainer with Right Timing of the Foot Trajectory
}

\author{
Fu-Chen Chen ${ }^{1, *}$, Yih-Fong Tzeng ${ }^{2}$, Meng-Hui Hsu ${ }^{1}$ \\ ${ }^{1}$ Department of Mechanical Engineering, Kun Shan University, Tainan, Taiwan \\ ${ }^{2}$ Department of Mechatronics Engineering, National Kaohsiung University of Science and Technology, Kaohsiung, Taiwan \\ Received 05 March 2020; received in revised form 06 May 2020; accepted 10 June 2020 \\ DOI: https://doi.org/10.46604/aiti.2020.5645
}

\begin{abstract}
The existing elliptical trainer cannot provide the user with the real jogging exercising mode and does not meet the principles of ergonomics. The purpose of this paper is to propose and study an innovative elliptical trainer that imitates the right timing of the foot trajectory while jogging. First of all, this study proposes and illustrates the structure and function of the innovative elliptical trainer with quick-return effect. Then, by using vector-loop method and motion geometry of the mechanism, the proposed innovative mechanism is studied kinematically. A design example is presented for interpreting the design process. At last, the foot trajectory of the innovative elliptical trainer is analyzed and confirmed. The simulation results confirm that the timing of the foot trajectory of the foot support members satisfies the principles of ergonomics, and keeps the user's legs from injury.
\end{abstract}

Keywords: innovative design, mechanism design, kinematics, elliptical trainer

\section{Introduction}

Jogging is a popular exercise, but it is known that the jogger's knees may suffer from significant impact especially at the time when the user's foot hits the ground. The knees could be injured after constantly taking the impacts for a period of time. It has been estimated that between $65-70 \%$ of runners will suffer an overuse injury in their lower extremities [1]. About 30 million Americans run for recreation or competition. Each year between 1/4 and 1/2 of runners lead to serious injury and cause a change in exercise or training [2-4]. Therefore, the elliptical trainer is developed to guide the users' feet to follow a track such that impact forces are minimized and the knees are well protected from being injured. Research on the lower extremity movements and forces generated during exercise on the elliptical trainer have demonstrated the elliptical motion which produces lower impact forces than treadmill running during elliptical exercise and walking [5-7].

There are many patents about elliptical trainers, but few researches are studied on the mechanism design of elliptical trainer. Shyu et al. [8] presented a novel design of adjustable elliptical trainer and the parameters that affect the elliptical path and inclined angle of the foot trajectory are investigated. Knutzen et al. [9] studied the influence of ramp position on joint biomechanics with adjusting the ramp setting during elliptical trainer exercise. Although quite a few kinematic structures have been used on the design of elliptical trainers, few of these apparatuses give pedal paths that might boost the lower extremity kinematics of gaits near the ground. Nelson and Burnfield [10] proposed a novel design method for elliptical exercisers and created a foot trajectory that more closely mimics the lower extremity kinematics of gait. This design includes the substitution

* Corresponding author. E-mail address:fcchen@ mail.ksu.edu.tw

Tel.: +886-6-2050496; Fax: +886-6-2050509 
of a modified Cardan gear system for the typical crank. Yin [11] used ADAMS to simulate the dynamics of elliptical exerciser and accurately describe its performance and the characteristic curves of some major components.

On conventional elliptical trainer, the joints connecting the flywheel to two supporting members are placed on the flywheel with a $180^{\circ}$ phase angle. Therefore, when one of the user's feet is at the front end of a pedal trajectory and about to support the user's weight, the other is at the rear end of the pedal trajectory as shown in Fig. 1(a). In other words, the supporting travel A1 and the striding travel A2 of the pedal trajectory A have almost the same path length.

Critics of the elliptical trainer have expressed concerns that the foot trajectory seems unergonomic and could cause the knee to harmful loads, resulting in injury [4]. The foot trajectories during treadmill walking or running are tracked by using webcam technology [12] and wearable wireless ultrasonic sensor network [13]. The foot trajectory of real jogging is shown in Fig. 1(b). When one of the user's feet is at the front end of the trajectory and starts supporting the user's weight, the other one has not yet reached the rear end of the trajectory but still on the way of its backward path. In fact, it could not begin to move forward until it reaches the rear end of the trajectory. As shown in Fig. 1(b), the path length of the supporting travel B1 should be shorter than that of the striding travel B2. As a result, the conventional elliptical trainer cannot provide the user with the real jogging exercising mode and does not meet the principles of ergonomics.

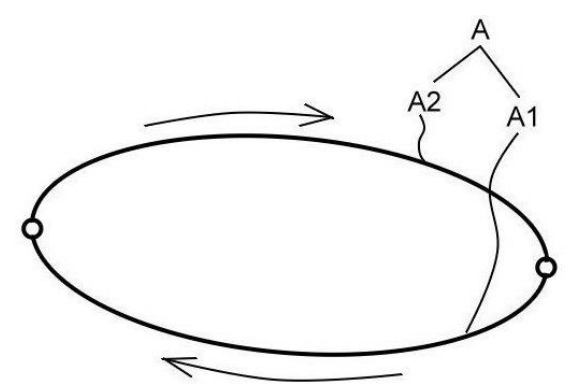

(a) conventional trajectory

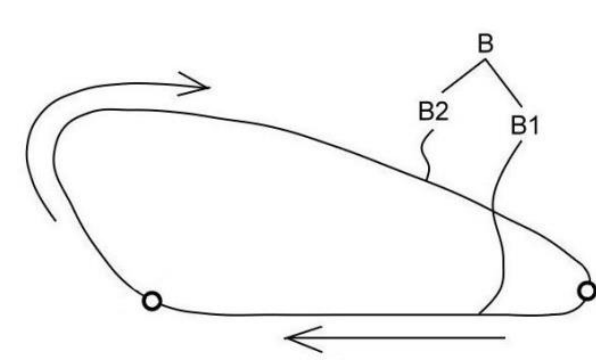

(b) real jogging trajectory

Fig. 1 Foot trajectory

Quick return mechanisms can be seen in every corner of engineering industry in various machines such as shaper, stamping press, power-driven reciprocating saw and so on. Several structures of the quick-return mechanisms can be found [14-15], including crank-shaper mechanisms, Whitworth mechanism and offset crank-slider mechanism. Quick-return mechanisms are usually used in machine tools for the intention of providing the reciprocating cutting tool a slow cutting stroke and a quick return stroke with a constant angular speed of the driving flywheel. The ratio of the time required for the cutting stroke to the time required for the return stroke is called the time ratio (TR) and is greater than unity [15].

The study of quick-return mechanisms has been investigated by quite a few researchers, and many important contributions have been accomplished. Dwivedi [16] modified the Whitworth quick-return mechanism to construct a high-velocity impacting press. The impacting press machine includes a Whitworth quick-return mechanism comprising a crank and a drive arm together with a variable speed D.C. motor, and a flywheel. This study also analyzes the reasons of the unbalanced forces of this high velocity machine and manages to reduce the forces delivered to the foundations.

Fung et al. [17-18] derived the governing equation of a quick-return mechanism by using the finite element method (FEM) with time-dependent length and Hamilton's principle. Hsieh and Tsai [19] proposed a novel design for quick-return mechanism that combines a generalized Oldham coupling and a slider-crank mechanism. The proposed quick-return mechanism is more compact and can be balanced easier than a conventional design. Chen et al. [20] proposed an elliptical trainer that composes of a conventional elliptical trainer and a draglink mechanism to generate a quick-return effect in order to mimic the timing of the foot trajectory while jogging. But the design procedures are too complicated, it is difficult for the designers to adjust the dimension to meet the timing of the foot trajectory while jogging. 
The purpose of this study is to propose an innovative elliptical trainer that takes advantage of an inverted slider-crank mechanism to imitate the timing of the pedal trajectory and study the kinematics of the design. By using the inverted slider-crank mechanism, the design procedures are simple and easy to adjust the dimension to meet the requirement of time ratio in the proposed design.

\section{Innovative Elliptical Trainer}

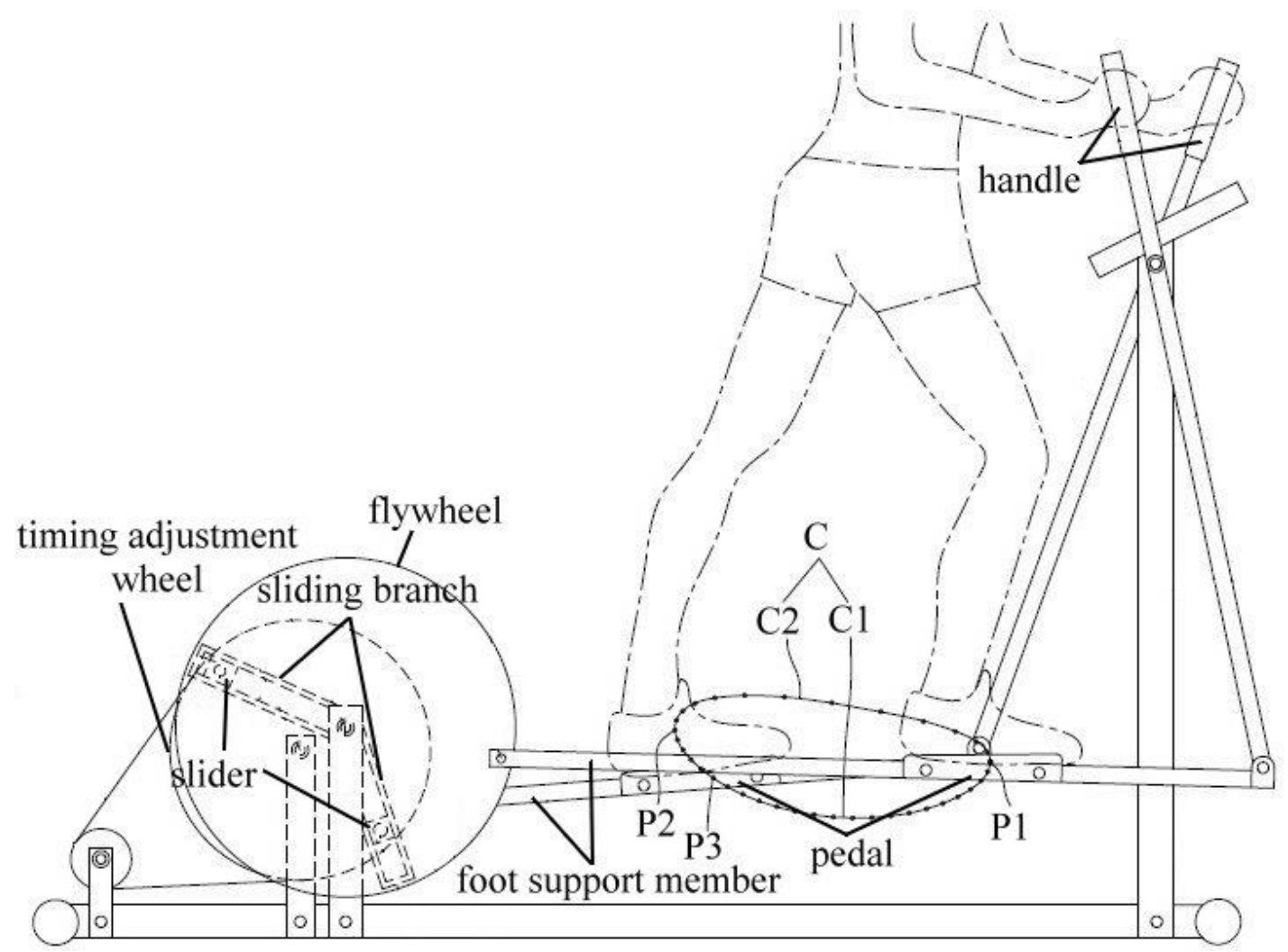

(a) innovative design

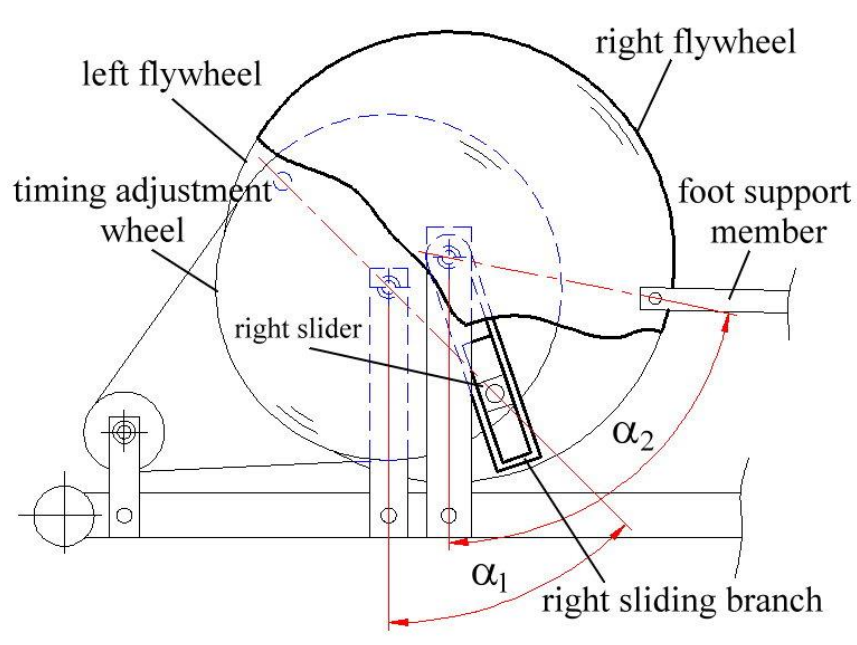

(b) detail drawing when right foot at a front end

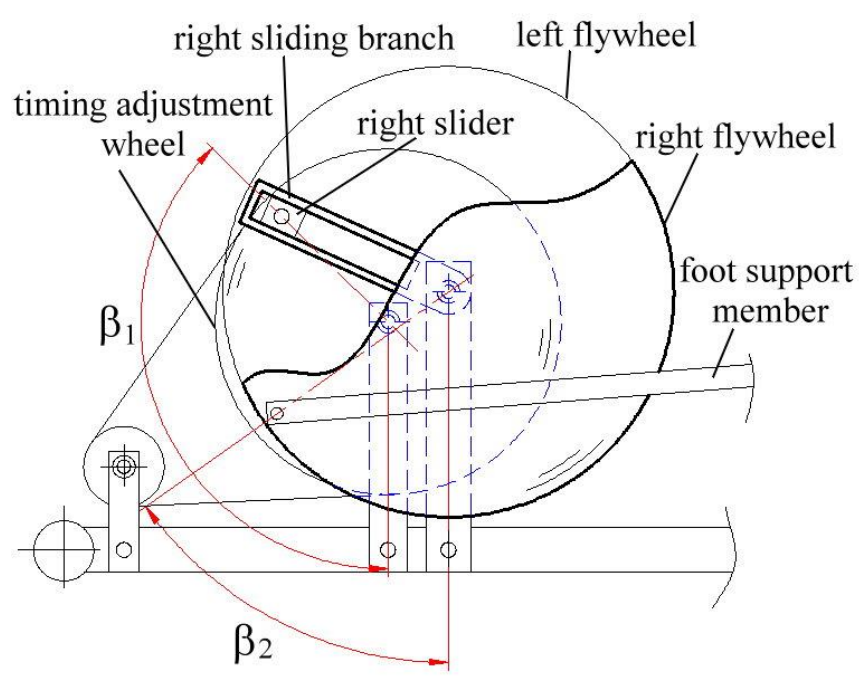

(c) detail drawing when right foot at a rear end

Fig. 2 Innovative elliptical trainer

The innovative elliptical trainer proposed in this study is illustrated in Fig. 2. The design comprises a frame, two flywheels, two swing handles, two foot support members, two sliders and a timing adjustment wheel. A sliding branch is created on each of the flywheels. Each of the foot support members connects to a respective flywheel. When the flywheel rotates about its pivot, the foot support member moves along a closed pedal trajectory $\mathrm{C}$. The pedal trajectory $\mathrm{C}$ comprises a supporting travel $\mathrm{C} 1$ (from P1 to P3) and a striding travel C2 (from P3 to P1). The timing adjustment wheel rotates about a fixed pivot on the frame. 
A right slider sliding along the sliding branch on the right flywheel connects to the right side of the timing adjustment wheel. On the contrary, a left slider sliding along the sliding branch on the left flywheel connects to the left side of the timing adjustment wheel. The right and left sliders are connected to the same timing adjustment wheel only with $180^{\circ}$ phase angle.

The motion of the elliptical trainer is described as follows. Fig. 2 shows that a user stands on the two foot support members, where the right foot support member is pedaled by the user's right foot and located at a front end P1, and the left one is pedaled by the user's left foot and located at a point P3. Accordingly, when the foot support member pedaled by the user's right foot is pedaled downward, the right flywheel with the sliding branch is driven to rotate about its fixed pivot. Therefore, the timing adjustment wheel is driven to rotate according to the relative movement between the right slider and the sliding branch of the right flywheel. Then, the timing adjustment wheel drives the left flywheel with the sliding branch to rotate according to the relative movement between the left slider and the sliding branch of the left flywheel so as to guide the left foot support member pedaled by the user's left foot to move upward. Furthermore, when the foot support member pedaled by the user's right foot finishes the supporting travel $\mathrm{C} 1$ and reaches the point P3, the foot support member pedaled by the user's left foot is guided to finish the striding travel $\mathrm{C} 2$ and reaches the front end P1.

The quick-return effect is further explained as follows. When the timing adjustment wheel rotates $180^{\circ}$ clockwise $\left(a_{1}+\beta_{1}=180^{\circ}\right.$ in Fig. 2(b) and 2(c)), the respective angle that the right flywheel rotates is less than $180^{\circ}\left(a_{2}+\beta_{2}=\right.$ $180^{\circ}$ in Fig. 2(b) and 2(c)). Therefore, the rotational speed of the timing adjustment wheel is faster than that of the right flywheel when the foot support member pedaled by the user's right foot moves within the supporting travel $\mathrm{C}$. The foot support member pedaled by the user's right foot is located at the point P3 rather than at the rear end P2 when the foot support members pedaled by the user's left foot just reaches the front end P1 of the pedal trajectory C. As the user's left foot reaches the supporting travel C1, the user's right foot begins to lift backward and upward. As a result, the user can shift his weight from one leg to the other before both his two legs are extended to their respective extreme positions so that the user is protected from muscle sore and pain. The timing of pedal trajectory $\mathrm{C}$ generated by the proposed elliptical trainer is closer to that in the real jogging and meets the principles of ergonomics.

\section{Motion Geometry of the Quick-Return Effect}

The skeleton drawing of the proposed innovative elliptical trainer assembly is shown in Fig. 3. From the vector loop diagram of the innovative elliptical trainer mechanism in Fig. 4, the following two vector loop equations can be derived as:

$$
\begin{aligned}
& \vec{r}_{2}-\vec{r}_{4}-\vec{r}_{1}=0 \\
& \vec{r}_{5}+\vec{r}_{6}-\vec{r}_{7}-\vec{r}_{8}-\vec{r}_{9}=0
\end{aligned}
$$

Decomposing the vectors in Eq. (2) into $X$ and $Y$ scalar components, the equations become

$$
\begin{aligned}
& r_{5} \cos \theta_{5}+r_{6} \cos \theta_{6}-r_{7} \cos \theta_{7}-r_{8}=0 \\
& r_{5} \sin \theta_{5}+r_{6} \sin \theta_{6}-r_{7} \sin \theta_{7}-r_{9}=0
\end{aligned}
$$

where $\theta_{i}$ is the position angle of vector $\bar{r}_{i}$ and the angle is defined positive when measured counterclockwise. When the foot support member is at the front end P1 of the pedal trajectory in Fig. 2, the position angles of flywheel and foot support link are the same (as shown in Figs. 3 and 5(a)), i.e. $\theta_{5}=\theta_{6}$. Substituting this into Eqs. (3) and (4) yields

$$
\begin{aligned}
& \left(r_{5}+r_{6}\right) \cos \theta_{5}=r_{7} \cos \theta_{7}+r_{8} \\
& \left(r_{5}+r_{6}\right) \sin \theta_{5}=r_{7} \sin \theta_{7}+r_{9}
\end{aligned}
$$

Squaring Eqs. (5) and (6) and adding them together yields 


$$
A \cos \theta_{7}+B \sin \theta_{7}+C=0
$$

where

$$
\begin{aligned}
& A=2 r_{7} r_{8} \\
& B=2 r_{7} r_{9} \\
& \mathrm{C}=r_{7}^{2}+r_{8}^{2}+r_{9}^{2}-\left(r_{5}+r_{6}\right)^{2}
\end{aligned}
$$

By solving Eq. (7), the position angle of the handle, $\theta_{7}$, is obtained as:

$$
\theta_{7}=2 \tan ^{-1}\left(\frac{-B \pm \sqrt{A^{2}+B^{2}-C^{2}}}{C-A}\right)
$$

From Eqs. (5) and (6), the angle of the flywheel, $\theta_{5}$, can be expressed as:

$$
\theta_{5}=\tan ^{-1} \frac{r_{7} \sin \theta_{7}+r_{9}}{r_{7} \cos \theta_{7}+r_{8}}
$$

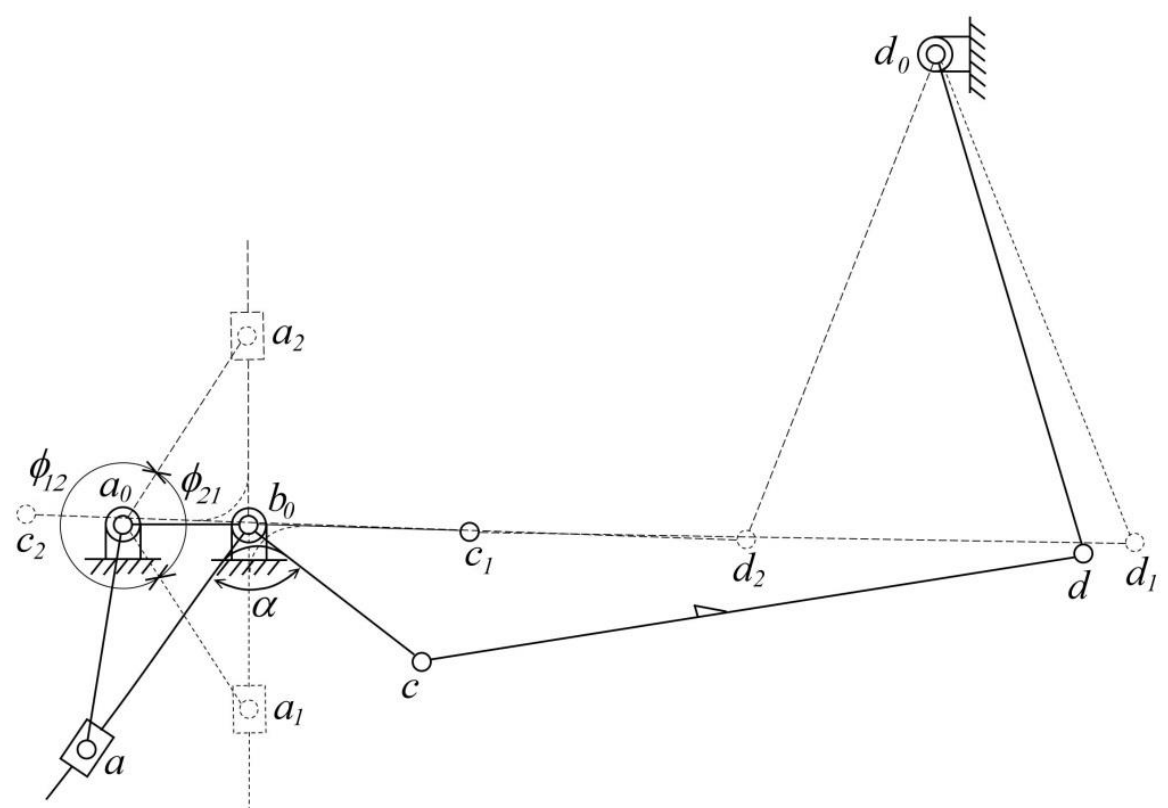

Fig. 3 Skeleton drawing of the innovative elliptical trainer

Assume that the angle between the flywheel and the sliding branch is $a$, as shown in Figs. 3-5. When the foot supporting member is at the front end $\mathrm{P} 1$ of the pedal trajectory (position $a_{0} a_{1} b_{0} c_{1} d_{1} d_{0}$ ) in Fig. 3, the position angle of sliding branch, $\theta_{4}$, can be found by

$$
\theta_{4}=\theta_{5}-\alpha
$$

By decomposing the vectors in Eq. (1) into $X$ and $Y$ scalar components and rearranging them yields

$$
\begin{aligned}
& r_{2} \cos \theta_{2}=r_{4} \cos \theta_{4}+r_{1} \\
& r_{2} \sin \theta_{2}=r_{4} \sin \theta_{4}
\end{aligned}
$$

Squaring Eqs. (14) and (15) and adding them together yields

$$
r_{4}^{2}+\left(2 r_{1} \cos \theta_{4}\right) r_{4}+r_{1}^{2}-r_{2}^{2}=0
$$




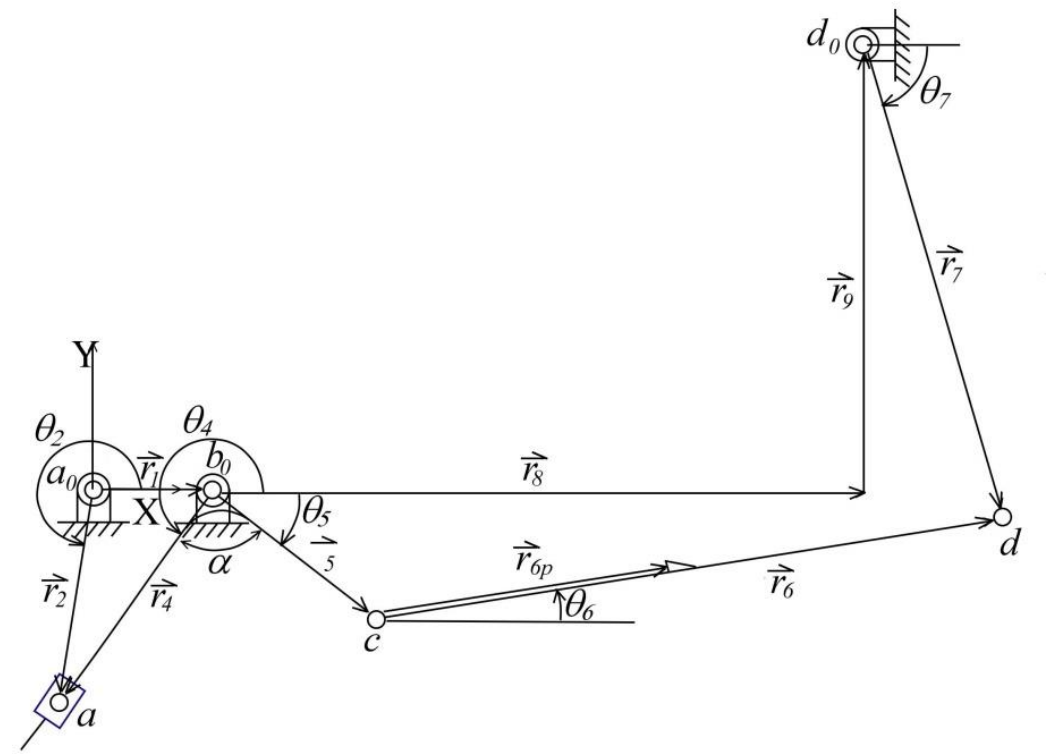

Fig. 4 Vector loop diagram

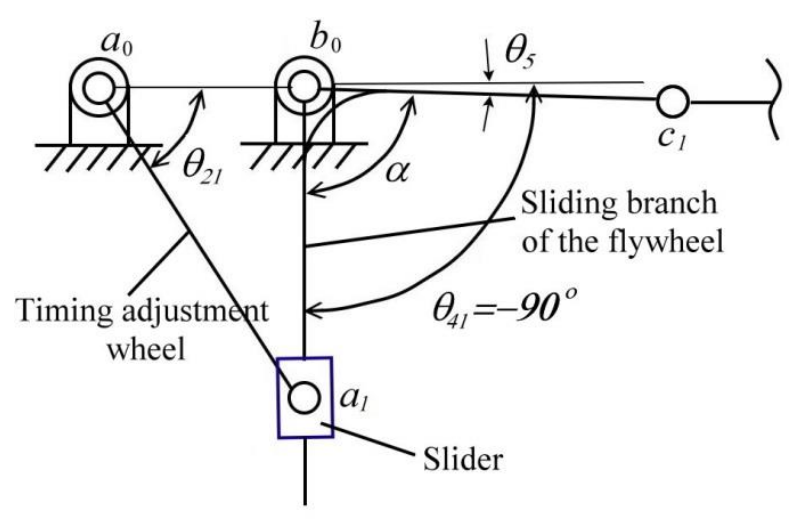

(a) right foot at a front end

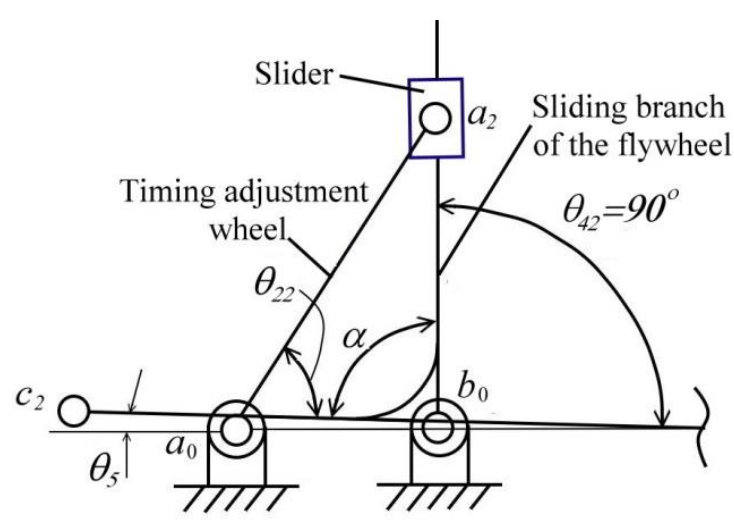

(b) right foot at a rear end

Fig. 5 Detail drawing of inverted slider-crank mechanism

By solving Eq. (16), the length of vector, $r_{4}$, is obtained as:

$$
r_{4}=-r_{1} \cos \theta_{4} \pm \sqrt{\left(r_{1} \cos \theta_{4}\right)^{2}-\left(r_{1}^{2}-r_{2}^{2}\right)}
$$

From Eqs. (14) and (15), the position angle of the timing adjustment wheel, $\theta_{2}$, can be expressed as:

$$
\theta_{2}=\tan ^{-1} \frac{r_{4} \sin \theta_{4}}{r_{4} \cos \theta_{4}+r_{1}}
$$

Similarly, when the foot support member is at the rear end P2 of the pedal trajectory (position $a_{0} a_{2} b_{0} c_{2} d_{2} d_{0}$ ) in Fig. 3, the difference between the position angles of flywheel and foot support link is $180^{\circ}$ (as shown in Figs. 3 and $5(\mathrm{~b})$ ), i.e. $\theta_{6}=\theta_{5}-$ $\pi$. Substituting this into Eqs. (3) and (4) and yields

$$
\begin{aligned}
& \left(r_{5}-r_{6}\right) \cos \theta_{5}=r_{7} \cos \theta_{7}+r_{8} \\
& \left(r_{5}-r_{6}\right) \sin \theta_{5}=r_{7} \sin \theta_{7}+r_{9}
\end{aligned}
$$

Squaring Eqs. (19) and (20) and adding them together yields

$$
A \cos \theta_{7}+B \sin \theta_{7}+C=0
$$

where 


$$
\begin{aligned}
& A=2 r_{7} r_{8} \\
& B=2 r_{7} r_{9} \\
& \mathrm{C}=r_{7}^{2}+r_{8}^{2}+r_{9}^{2}-\left(r_{5}-r_{6}\right)^{2}
\end{aligned}
$$

By solving Eq. (21), the position angle of the handle, $\theta_{7}$, is obtained as:

$$
\theta_{7}=2 \tan ^{-1}\left(\frac{-B \pm \sqrt{A^{2}+B^{2}-C^{2}}}{C-A}\right)
$$

From Eqs. (19) and (20), the angle of the flywheel, $\theta_{5}$, can be expressed as:

$$
\theta_{5}=\tan ^{-1} \frac{r_{7} \sin \theta_{7}+r_{9}}{r_{7} \cos \theta_{7}+r_{8}}
$$

As the foot support member is at the rear end P2 of the pedal trajectory in Fig. 3, the position angle of the sliding branch, $\theta_{4}$, can be obtained from Eq. (13). Since the position angle of $\theta_{4}$ is known, the length of vector $\vec{r}_{4}, r_{4}$, and the position angle of the timing adjustment wheel, $\theta_{2}$, can be obtained from Eqs. (17) and (18) as well.

Assume that $\emptyset_{12}$ is the angular displacement of the flywheel as the pedal trajectory moves from the front end P1 to the rear end $\mathrm{P} 2$, and $\emptyset_{21}$ is from the rear end P2 to the front end P1. The summation of these two angles is $360^{\circ}$, that is

$$
\phi_{12}+\phi_{21}=2 \pi
$$

Time ratio $(T R)$ is the ratio of the time required for the slow stroke to the time required for the quick stroke. If the timing adjustment wheel runs at constant velocity, the time ratio $T R$ can be expressed as:

$$
T R=\frac{\phi_{12}}{\phi_{21}}
$$

Assume the time ratio is known, then the rotation angles of the two strokes of the foot trajectory between two extreme positions can be expressed as:

$$
\begin{aligned}
& \phi_{21}=\frac{2 \pi}{1+T R} \\
& \phi_{12}=2 \pi-\phi_{21}
\end{aligned}
$$

As the pedal trajectory moves from the front end P1 to the rear end P2, the flywheel rotates with respect the fixed pivot and the rotation angle is approximately $180^{\circ}$. When the pedal position is at the front end $\mathrm{P} 1$, assume that the position angle of the sliding branch (link 4) is perpendicular downwards as shown in Fig. 5(a). At this time, $\theta_{41}$ denotes the position angle of the sliding branch and is equal to $-90^{\circ}$ while the position angle of timing adjustment wheel (link 2) is denoted as $\theta_{21}$. When the pedal position is at the rear end P2, assume that the position angle of the sliding branch is perpendicular upwards as shown in Fig. 5(b). At this time, $\theta_{42}$ denotes the position angle of the sliding branch and is equal to $90^{\circ}$ while the position angle of timing adjustment wheel is denoted as $\theta_{22}$. From the geometry of the inverted slider-crank mechanism in Fig. 5, the position angles of the timing adjustment wheel, $\theta_{21}$ and $\theta_{22}$, are symmetrical. Therefore, $\theta_{22}=-\theta_{21}$ and the lengths of vector $\vec{r}_{4}$ at these two position are the same. Furthermore, $\emptyset_{21}$ can be expressed as:

$$
\phi_{21}=\theta_{22}-\theta_{21}=-\theta_{21}=\theta_{22}
$$

When the time ratio $(T R)$ is known, the position angle of the timing adjustment wheel can be calculated from Eqs. (29) and (31). 
Furthermore, when the pedal position is at the front end P1 and the position of the sliding branch is downwards, i.e., $\theta_{41}=-90^{\circ}$, the position angle of timing adjustment wheel $\theta_{2}$ is equal to $\theta_{21}$. It can be observed that there are three unknown variables $\left(r_{1}, r_{2}, r_{4}\right)$ in Eqs. (14) and (15). Assume that the length of the fixed link, $r_{1}$, is known, the length of the link $2, r_{2}$, can be obtained as:

$$
r_{2}=\frac{r_{1}}{\cos \theta_{21}}
$$

As the pedal trajectory moves from the front end P1 to the rear end P2, the flywheel rotates with respect to the fixed pivot and the rotation angle of the flywheel is approximately $180^{\circ}$. According to the assumption above, the relationship between the stroke angles $\left(\emptyset_{12}\right.$ and $\left.\emptyset_{21}\right)$ and the length ratio $\left(r_{2} / r_{1}\right)$ of the timing adjustment wheel to the fixed link is shown in Fig. 6(a). Fig. 6(a) shows the stroke angle $\emptyset_{12}$ is larger than the stroke angle $\emptyset_{21}$. The smaller the length ratio is, the larger the stroke angle $\emptyset_{12}$ is and the smaller the stroke angle $\emptyset_{21}$ is. However, when the length ratio of the timing adjustment wheel to the fixed link is increased, the difference between the stroke angles $\emptyset_{12}$ and $\emptyset_{21}$ is reduced. The relationship between the time ratio and the length ratio is shown in Fig. 6(b). From Fig. 6(b), the smaller the length ratio of the timing adjustment wheel to the fixed link is used, the larger the time ratio becomes. On the contrary, the larger the length ratio is used, the smaller the time ratio becomes. By using Fig. 6(b), it can be used to select proper length ratio to generate desired time ratio.

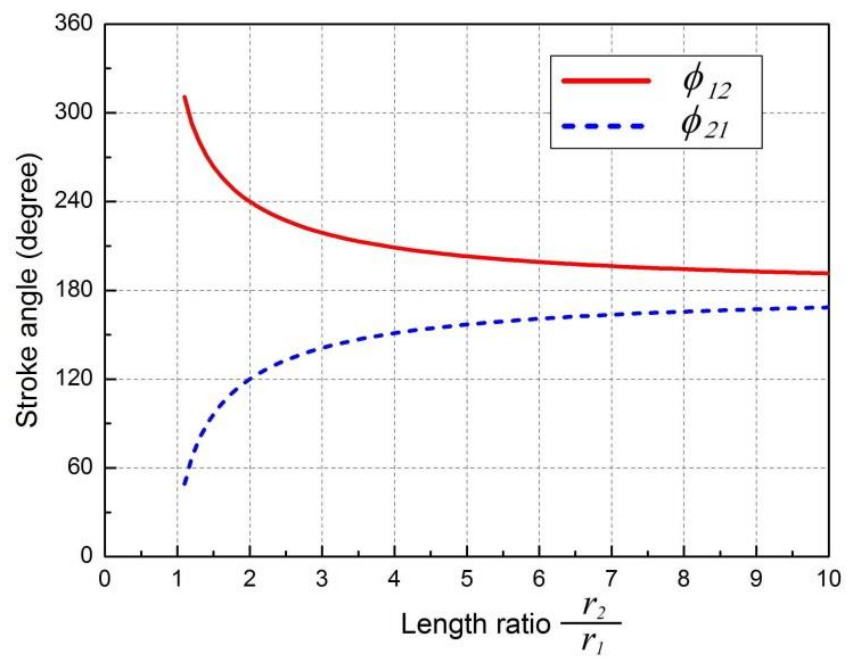

(a) stroke angles

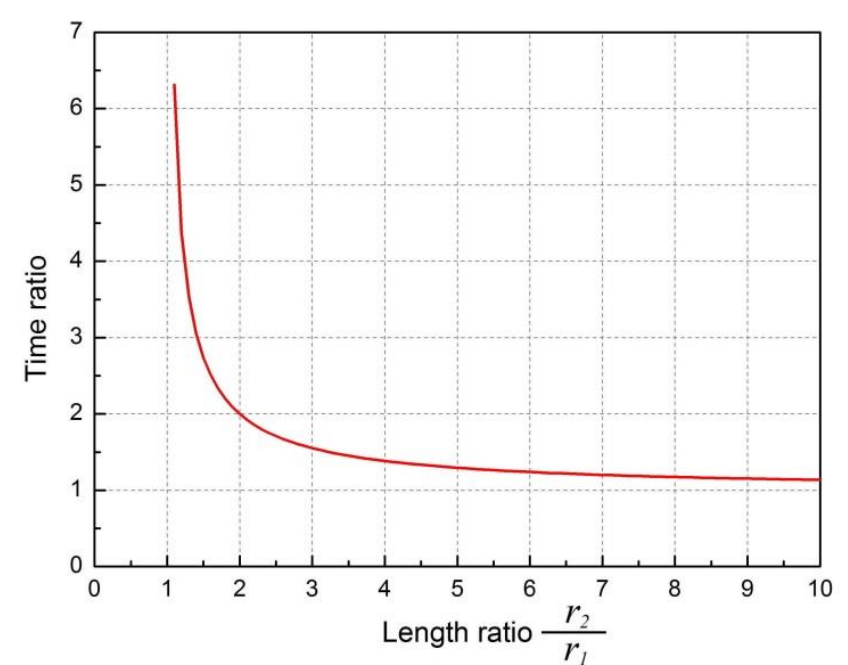

(b) time ratio

Fig. 6 Stroke angles and time ratio

\section{Kinematic Analysis}

When the dimensions of the elliptical trainer mechanism and the position angle of the timing adjusting wheel $\theta_{2}$ are known, the complete kinematic analysis and pedal trajectory can be derived and calculated. From Eqs. (14) and (15), the position angle of the sliding branch, $\theta_{4}$, can be expressed as:

$$
\theta_{4}=\tan ^{-1} \frac{r_{2} \sin \theta_{2}}{r_{2} \cos \theta_{2}-r_{1}}
$$

By expressing the Eq. (2) into $X$ and $Y$ scalar component equations and rearranging, the equations become

$$
\begin{aligned}
& r_{6} \cos \theta_{6}=r_{7} \cos \theta_{7}+r_{8}-r_{5} \cos \theta_{5} \\
& r_{6} \sin \theta_{6}=r_{7} \sin \theta_{7}+r_{9}-r_{5} \sin \theta_{5}
\end{aligned}
$$

where $\theta_{5}=\theta_{4}+\alpha$. Squaring Eqs. (34) and (35) and adding them together yields 


$$
A \cos \theta_{7}+B \sin \theta_{7}+C=0
$$

where

$$
\begin{aligned}
& \mathrm{A}=2 r_{7}\left(r_{8}-r_{5} \cos \theta_{5}\right) \\
& \mathrm{B}=2 r_{7}\left(r_{9}-r_{5} \sin \theta_{5}\right) \\
& \mathrm{C}=r_{5}^{2}-r_{6}^{2}+r_{7}^{2}+r_{8}^{2}+r_{9}^{2}-2 r_{5} r_{8} \cos \theta_{5}-2 r_{5} r_{9} \cos \theta_{5}
\end{aligned}
$$

By solving Eq. (36), the position angle of the handle, $\theta_{7}$, is obtained as:

$$
\theta_{7}=2 \tan ^{-1}\left(\frac{-B \pm \sqrt{A^{2}+B^{2}-C^{2}}}{C-A}\right)
$$

From Eqs. (34) and (35), the angle of foot support link, $\theta_{6}$, can be found by

$$
\theta_{6}=\tan ^{-1} \frac{r_{7} \sin \theta_{7}+r_{9}-r_{5} \sin \theta_{5}}{r_{7} \cos \theta_{7}+r_{8}-r_{5} \cos \theta_{5}}
$$

Differentiating Eqs. (14) and (15) with respect to time yields velocity equations as:

$$
\begin{aligned}
& -\left(r_{2} \sin \theta_{2}\right) \dot{\theta}_{2}+\left(r_{4} \sin \theta_{4}\right) \dot{\theta}_{4}-\left(\cos \theta_{4}\right) \dot{r}_{4} \\
& -\left(r_{2} \cos \theta_{2}\right) \dot{\theta}_{2}+\left(r_{4} \cos \theta_{4}\right) \dot{\theta}_{4}-\left(\sin \theta_{4}\right) \dot{r}_{4}
\end{aligned}
$$

When the velocity of timing adjusting wheel $\dot{\theta}_{4}$ is known. By using Gauss-Jordan method to solve Eqs. (42) and (43), $\dot{\theta}_{4}$ and $\dot{r}_{4}$ can be obtained as:

$$
\begin{aligned}
& \dot{\theta}_{4}=\frac{r_{2} \cos \left(\theta_{2}-\theta_{4}\right)}{r_{4}} \dot{\theta}_{2} \\
& \dot{r}_{4}=-r_{2} \sin \left(\theta_{2}-\theta_{4}\right) \dot{\theta}_{2}
\end{aligned}
$$

Differentiating Eqs. (42) and (43) with respect to time yields velocity equations as:

$$
\begin{aligned}
& -\left(r_{6} \sin \theta_{6}\right) \dot{\theta}_{6}+\left(r_{7} \sin \theta_{7}\right) \dot{\theta}_{7}=\left(r_{5} \sin \theta_{5}\right) \dot{\theta}_{5} \\
& -\left(r_{6} \cos \theta_{6}\right) \dot{\theta}_{6}-\left(r_{7} \cos \theta_{7}\right) \dot{\theta}_{7}=-\left(r_{5} \cos \theta_{5}\right) \dot{\theta}_{5}
\end{aligned}
$$

where $\dot{\theta}_{5}=\dot{\theta}_{4}$. By using Gauss-Jordan method to solve Eqs. (46) and (47), $\dot{\theta}_{6}$ and $\dot{\theta}_{7}$ can be obtained as:

$$
\begin{aligned}
& \dot{\theta}_{4}=\frac{r_{5} \sin \left(\theta_{5}-\theta_{7}\right)}{r_{6} \sin \left(\theta_{7}-\theta_{6}\right)} \dot{\theta}_{5} \\
& \dot{\theta}_{7}=\frac{r_{5} \sin \left(\theta_{5}-\theta_{6}\right)}{r_{6} \sin \left(\theta_{7}-\theta_{6}\right)} \dot{\theta}_{5}
\end{aligned}
$$

Therefore, the pedal position $\left(x_{p}, y_{p}\right)$ with respect to the fixed pivot of the flywheel can be expressed as:

$$
\begin{aligned}
& x_{p}=r_{5} \cos \theta_{5}+r_{6 p} \cos \theta_{6} \\
& y_{p}=r_{5} \sin \theta_{5}+r_{6 p} \sin \theta_{6}
\end{aligned}
$$

Differentiating Eqs. (50) and (51) with respect to time yields the pedal velocity as: 


$$
\begin{aligned}
& \dot{x}_{p}=-r_{5} \sin \theta_{5} \dot{\theta}_{5}-r_{6 p} \sin \theta_{6} \dot{\theta}_{6} \\
& \dot{y}_{p}=r_{5} \cos \theta_{5} \dot{\theta}_{5}+r_{6 p} \cos \theta_{6} \dot{\theta}_{6}
\end{aligned}
$$

\section{Design Example and Discussion}

To explain the design procedure of the proposed innovative mechanism, a design example is presented here for illustration. Referring to a conventional design, assume that the parameters $r_{5}, r_{6}, r_{7}, r_{8}$ and $r_{9}$ are $28.0 \mathrm{~cm}, 134.0 \mathrm{~cm}, 93.0 \mathrm{~cm}$, $143.0 \mathrm{~cm}$ and $82.0 \mathrm{~cm}$ respectively. Using Eqs. (11) and (12), the angles $\theta_{7}$ and $\theta_{5}$ are calculated as $\theta_{7}=-78.37^{\circ}$ and $\theta_{5}=-3.22^{\circ}$ respectively. Assume that the position angle of the sliding branch is $\theta_{4}=\theta_{41}-90^{\circ}$ when the foot support member is at the front end of the pedal trajectory. The angle between the flywheel and the sliding branch can be calculated from Eq. (13) as $\alpha=\theta_{5}-\theta_{4}=-86.78^{\circ}$.

Assume that the time ratio $(T R)$ is 2.0 , the angles $\emptyset_{21}=120^{\circ}, \emptyset_{12}=240^{\circ}$ and $\theta_{22}=-\theta_{21}=60^{\circ}$ can be obtained from Eqs. (29)-(31). Finally, assume that fixed length $r_{1}$ is $13.0 \mathrm{~cm}$, then the length of link 2 can be computed from Eq. (32) as $r_{2}=26.0 \mathrm{~cm}$.

By using the vector-loop method and the kinematic analysis, motion simulation of pedal trajectory is carried out to validate the feasibility of the proposed innovative mechanism. The pedal trajectories of a conventional and the proposed design are shown in Fig. 7. The pedal trajectories are drawn and dotted whenever the timing adjustment wheel is angular displaced with $10^{\circ}$ increment. In Fig. 7(a), for the conventional elliptical trainer, the distance between any adjacent points near the front and rear ends of the pedal trajectory is shorter and that in the middle of the supporting and striding travel is longer. In Fig. 7(b), for the proposed design, the distance between any adjacent points near the bottom of pedal trajectory is shorter and that near the top is longer. If the timing adjustment wheel is operated at a constant speed, the speed of the foot near the front and rear ends is slower and that in the middle of the supporting and striding travels is faster for the conventional elliptical trainer. However, the speed of the foot at the bottom of the pedal trajectory is slower and that at the top is quicker for the proposed design.

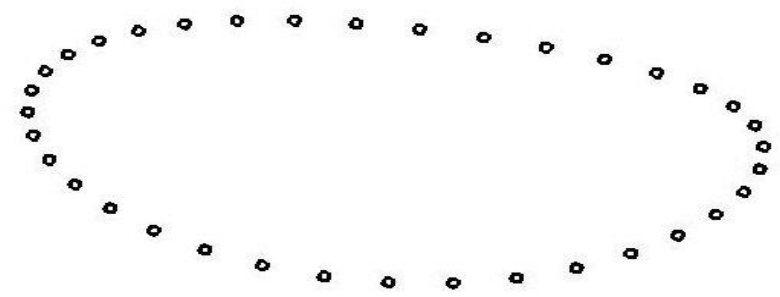

(a) conventional design

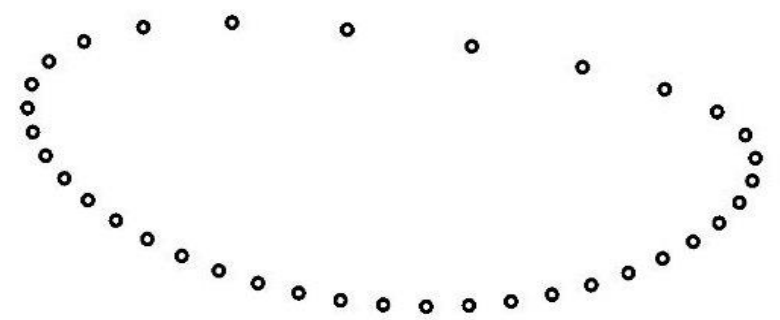

(b) proposed design

Fig. 7 Pedal trajectory

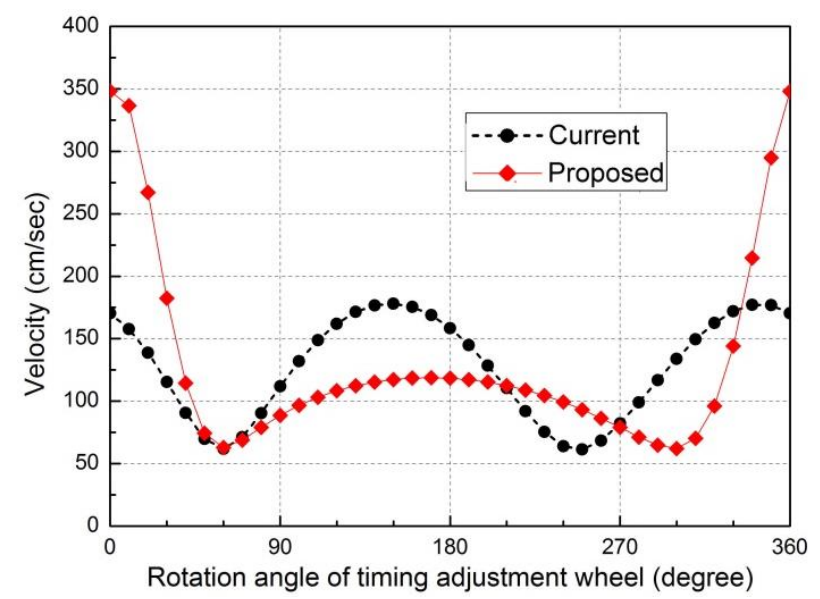

Fig. 8 Pedal velocity 
Assume that the timing adjustment wheel runs at $60.0 \mathrm{rpm}$, the velocity of the pedal on the foot support member is shown in Fig. 8. When the timing adjustment wheel is at about $60^{\circ}$ and $250^{\circ}$ of the conventional design, i.e. near the front and rear ends of trajectory, the pedal velocity is about $65.0 \mathrm{~cm} / \mathrm{sec}$. One the contrary, when the flywheel is at about $150^{\circ}$ and $340^{\circ}$, i.e. in the middle of the supporting and striding travels, the pedal velocity is about $175.0 \mathrm{~cm} / \mathrm{sec}$. The pedal velocity of the conventional design is not same as the real jogging.

However, when the timing adjustment rotates from $60^{\circ}$ to $300^{\circ}$ in the proposed design, i.e. near the bottom part of pedal trajectory, the pedal velocity changes between $65.0 \sim 120.0 \mathrm{~cm} / \mathrm{sec}$. When the flywheel is between $300^{\circ}$ and $60^{\circ}$, i.e. near the top part of pedal trajectory, the pedal velocity varies between $65.0 \sim 350.0 \mathrm{~cm} / \mathrm{sec}$. The maximum speed during the striding travel is 3 times than that during the supporting travel. Therefore, the average velocity of pedal trajectory on the supporting travel is slower than that on the striding travel. By using the quick-return effect of the inverted slider-crank mechanism, the timing of the pedal trajectory in this innovative design is more similar to the one in real jogging and meets the principles of ergonomics. When using this proposed innovative elliptical trainer design, the user can shift his body weight from one leg to the other before both of his legs extend to their extreme positions. Compared to the conventional design, such a motion pattern proposed in this study can protect the user from suffering muscle sore and pain. By using a 3D CAD design software, SolidWorks, the solid model of this innovative design is constructed and shown in Fig. 9. For the sake of showing the structure of the innovative design, the flywheel is drawn as a crank in Fig. 9. The CAD prototype can be used for assembly, simulation and fabrication in order to confirm its feasibility and expedite the commercialization.

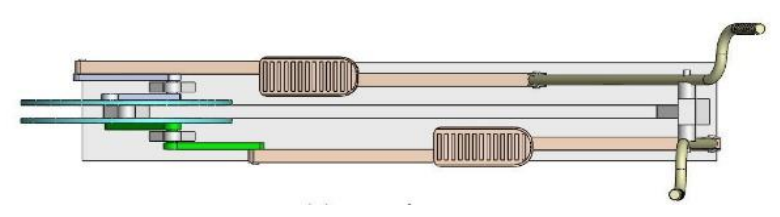

(a) top view

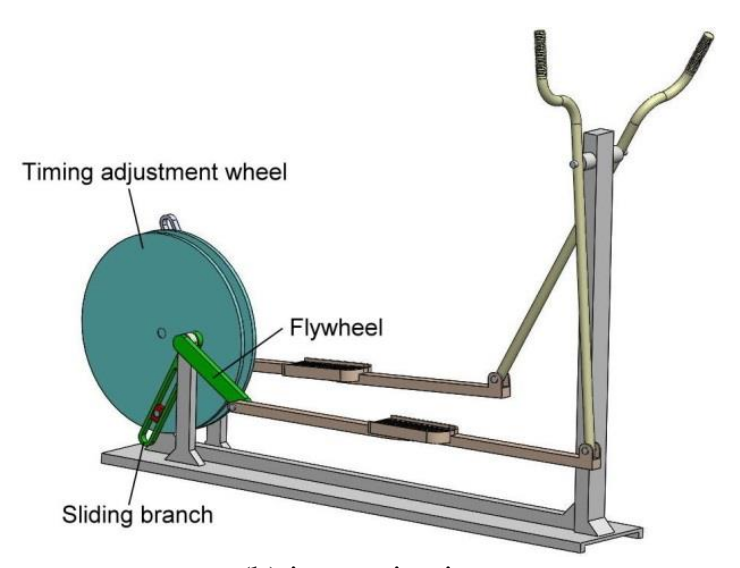

(b) isometric view

Fig. 9 CAD solid model of the innovative elliptical trainer

\section{Conclusions}

The purpose of this paper is to propose and study an innovative elliptical trainer that imitates the timing of the foot trajectory during jogging. The procedures for the design of elliptical trainer with required time-ratio are illustrated. The pedal trajectory and the kinematics of the innovative elliptical trainer are analyzed and simulated. The results of the simulation confirm that the velocity of pedal trajectory on the supporting travel in the proposed design is slower than that on the striding travel. Therefore, the proposed innovative design that mimics the timing of the foot trajectory can satisfy the design requirements and fit the principles of ergonomics for the joggers. Based on the results of this investigation, the foot trajectory and the workload of this design can be further modified by adjusting the dimension, the ramp setting and the resistance of the motion. As for the dynamics of the innovative elliptical trainer and its test data under ISO standard [21], they will be studied, collected and optimized in the future when the prototype is constructed.

\section{Conflicts of Interest}

The authors declare no conflict of interest. 


\section{References}

[1] J. H. Hoeberigs, "Factors related to the incidence of running injuries," Sports Medicine, vol.13, no. 6, pp. 408-422, November 1992.

[2] G. A. Hanks and A. Kalenak, Running injuries. In: Grana WA, Kalenak A, Editors. Clinical Sports Medicine. Philadelphia: W.B. Saunders Company, pp. 458-465, 1982.

[3] A. F. Renstrom, "Mechanism, diagnosis, and treatment of running injuries," Instructional Course Lectures, vol. 42, pp. 225-234, 1993.

[4] T. F. Novacheck, "The biomechanics of running," Gait and Posture, vol. 7, no. 1, pp. 77-95, January 1998.

[5] T. W. Lu, H. L. Chien, and H. L. Chen, "Joint Loading in the lower extremities during elliptical exercise," Medicine and Science in Sports and Exercise, vol. 39, no. 9, pp.1651-1658, September 2007.

[6] T. W. Lu and H. L. Chien, "Dynamic analysis of the lower extremities during elliptical exercise," Journal of Biomechanics, vol. 39, no. 1, p. S193, 2006.

[7] J. P. Porcari, J. M. Zedaker, L. Naser, and M. Miller, "Evaluation of an elliptical exerciser in comparison to treadmill walking and running, stationary cycling, and stepping," Medicine \& Science in Sports \& Exercise, vol. 30, no .5, p. 168, 1998.

[8] J. H. Shyu, C. K. Chen, C. C. Yu, and Y. J. Luo, "Research and development of an adjustable elliptical exerciser," Advanced Materials Research, vols. 308-310, pp. 2078-2083, August 2011.

[9] K. M. Knutzen, W. L. McLaughlin, A. J. Lawson, B. S. Row, and L. T. Martin, "Influence of ramp position on joint biomechanics during elliptical trainer exercise," The Open Sports Sciences Journal, vol. 3, pp. 165-177, 2010.

[10] C. A. Nelson and J. M. Burnfield, "Improved elliptical trainer biomechanics using a modified cardan gear," ASME 2012 International Design Engineering Technical Conferences and Computers and Information in Engineering Conference, vol. 4: 36th Mechanisms and Robotics Conference, August 2012, pp. 35-42.

[11] Z. Yin, "Modal analysis and dynamics simulation of elliptical trainer," International Conference on Mechatronics and Intelligent Robotics, March 2020, pp. 19-25.

[12] C. Krishnan, E. P. Washabaugh, and Y. Seetharaman, "A low cost real-time motion tracking approach using webcam technology," Journal of Biomechanics, vol. 48, no. 3, pp. 544-548, February 2015.

[13] Y. Qi, C. B. Soh, E. Gunawan, and K. S. Low, "Ambulatory measurement of three-dimensional foot displacement during treadmill walking using wearable wireless ultrasonic sensor network," IEEE Journal of Biomedical and Health Informatics, vol. 19, no. 2, pp. 446-452, March 2015.

[14] G. H. Martin, Kinematics and dynamics of machines, 2nd ed. USA: Waveland Press, 1982.

[15] H. S. Yan, Mechanisms: theory and applications, Singapore: McGraw-Hill, 2016.

[16] S. N. Dwivedi, "Application of a Whitworth quick return mechanism for high velocity impacting press," Mechanism and Machine Theory, vol. 19, no.1, pp. 51-59, 1984.

[17] R. F. Fung and K. W. Chen, "Constant speed control of the quick return mechanism driven by a dc motor," JSME International Journal Series C Mechanical Systems, Machine Elements and Manufacturing, vol. 40, no. 3, pp. 454-461, 1997.

[18] R. F. Fung and F. Y. Lee, "Dynamic analysis of the flexible rod of a quick-return mechanism with time-dependent coefficients by the finite element method," Journal of Sound and Vibration, vol. 202, no. 2, pp. 187-201, May 1997.

[19] W. H. Hsieh and C. H. Tsai, "A study on a novel quick return mechanism," Transactions of the Canadian Society for Mechanical Engineering, vol. 33, no. 3, pp. 487-500, March 2018.

[20] F. C. Chen, Y. F. Tzeng, and W. R. Chen, "On the mechanism design of an innovative elliptical exerciser with quick-return effect," International Journal of Engineering and Technology Innovation, vol. 8, no. 3, pp.228-239, July 2018.

[21] Stationary training equipment - Part 9: Elliptical trainers, additional specific safety requirements and test methods, ISO 20957-9, pp. 1-14, 2016.

Copyright $\odot$ by the authors. Licensee TAETI, Taiwan. This article is an open access article distributed under the terms and conditions of the Creative Commons Attribution (CC BY-NC) license (https://creativecommons.org/licenses/by-nc/4.0/). 